\title{
Ultrashort Implants, Alternative Prosthetic Rehabilitation in Mandibular Atrophies in Fragile Subjects: A Retrospective Study
}

\author{
Giovanni Falisi ${ }^{1}\left(\right.$, Carlo Di Paolo ${ }^{2}$, Claudio Rastelli ${ }^{1}$, Carlo Franceschini ${ }^{2}$, Sofia Rastelli ${ }^{1}$, Roberto Gatto ${ }^{1}$ \\ and Gianluca Botticelli ${ }^{1, *}$ \\ 1 Department of Life Health and Environmental Sciences, University of L'Aquila, 67100 L'Aquila, Italy; \\ giovanni.falisi@univaq.it (G.F.); claudio.rastelli@univaq.it (C.R.); sofiarastelli3@gmail.com (S.R.); \\ roberto.gatto@univaq.it (R.G.) \\ 2 Department of Oral and Maxillo-Facial Sciences, "Sapienza" University of Rome, 00185 Rome, Italy; \\ carlo.dipaolo@uniroma1.it (C.D.P.); franceschinicarlo93@gmail.com (C.F.) \\ * Correspondence: gianluca.botticelli@graduate.univaq.it
}

check for

updates

Citation: Falisi, G.; Di Paolo, C.;

Rastelli, C.; Franceschini, C.; Rastelli,

S.; Gatto, R.; Botticelli, G. Ultrashort

Implants, Alternative Prosthetic

Rehabilitation in Mandibular

Atrophies in Fragile Subjects:

A Retrospective Study. Healthcare

2021, 9, 175. https://doi.org/

10.3390/healthcare 9020175

Academic Editors: Takahiro Kanno

and Jitendra Singh

Received: 23 December 2020

Accepted: 3 February 2021

Published: 6 February 2021

Publisher's Note: MDPI stays neutral with regard to jurisdictional claims in published maps and institutional affiliations.

\begin{abstract}
This study aimed to evaluate the effectiveness of using ultrashort implants in the rehabilitation of jaws of fragile patients. The aim of the study was to retrospectively evaluate the survival rate of full-arch prosthetic rehabilitation on ultrashort implants, length $4 \mathrm{~mm}, 4 \mathrm{~mm}$ in diameter in the premolar and canine area and $4.5 \mathrm{~mm}$ in diameter in the molar area, with the insertion torque of $60 \mathrm{Nw}$ and immediate loading. Nineteen patients were evaluated for 3 years clinically and radiographically. The significant majority of the patients at the 3 year follow-up (T4) presented a stable and functional implant-supported prothesis, and the survival rate of the implants was $85 \%$, with a loss of 16 implants on 114 implants. The combination of the innovative implant surfaces and the correct project of the prostheses, with the related implant connection, determined a different timing in the therapy, allowing to obtain an immediate loading, which is currently demanded by patients. This and recent reports on short and ultrashort implant usage in atrophic jaws offer a good solution in critical cases. In conclusion, within the limits of the study, the full-arch rehabilitation with immediate loading on ultrashort implants showed good results with few postoperative complications and related low biological cost.
\end{abstract}

Keywords: ultrashort implants; immediate loading; full-arch rehabilitation; neutral zone

\section{Introduction}

The rehabilitation of edentulism in atrophic jaws represents a difficult situation to face, especially in fragile patients [1]. Indeed, the prosthetic treatment most required by patients is the implant-supported one [1].

The implant-supported prosthetic solutions require a bone quantity able to host the implant fixture and, in cases of atrophic jaws, ridge augmentation is required to place a fixture of "normal" length $(\leq 10 \mathrm{~mm})$ [2]. These kinds of interventions are not risk-free, and the placement of shorter lengths represents a valid alternative to rehabilitate atrophic jaws [3].

The definition of a short implant is still a topic of debate; some researchers define "short implants" as fixtures having a length ranging between 7 and $10 \mathrm{~mm}$, whereas others define them as fixtures presenting an intrabony length of $8 \mathrm{~mm}$ [4].

Indeed, some researchers consider $10 \mathrm{~mm}$ or less as short, while others propose $<8,<7$, or $6 \mathrm{~mm}$ as truly short $[5,6]$. Nevertheless, other researchers agree that ultrashort implants are $4 \mathrm{~mm}$ long [7-9].

Due to the biological and economic advantages in using this type of fixture to prosthetically rehabilitate an atrophic jaw, several studies reported at various follow-up points the survival rates of fixed prostheses implant supported, showing their efficacies [10-12]. 
Indeed, the consensus report of the Group 1 ITI (International team for Implantology) in 2018 evaluated the survival rates of short implants as similar to longer implants, with a lower rate of post-intervention complications if compared to the longer implants placed together with the procedure of bone grafting [13].

The success of the rehabilitation of an edentulism relies on the primary stability of the implant and on the correct project of the prosthesis, which should follow the masticatory dynamic of the patient, especially in full-arch rehabilitation [14].

In the Group 1 ITI consensus report, it is indeed stated the survival rate of short implants is influenced by the functionality.

Therefore, the construction of overdentures should be done with respect to the physiological state of the patient, known as the neutral zone, so that harmful masticatory forces do not affect the implants [15].

The combination of innovative implant surfaces and the correct project of the prostheses, with the related implant connection, determined a different timing in the therapy, allowing to obtain an immediate loading [16-18].

Immediate loading indeed relies on many factors, such as the number of implants and the biological bone response (mostly the primary stability) $[19,20]$.

In case of short implants, the improvement of the surfaces allows an optimal primary stability and, as a consequence, the possibility of immediate loading.

The aim of the study was to retrospectively evaluate at different timings the survival rate of full-arch prosthetic rehabilitation on ultrashort implants (length $4 \mathrm{~mm}$ ), with immediate loading.

\section{Materials and Methods}

\subsection{Patient Characteristics}

Between 2015 and 1027, approximately 150 patients that needed a total implantsupported inferior rehabilitation were examined at the Department of Odontostomatology of the University of L'Aquila. In order to achieve a successful prosthetic rehabilitation, all patients were studied through our new clinical protocol, which included a first gnathological evaluation through RC-TMD (Research Criteria- Temporomandibular Disorders) to evaluate the stomatognathic functional status [15].

The following exclusion criteria were applied for the selection of the study group: patients smoking more than 12 cigarettes per day; patients with high risk factors; patients on bisphosphonate therapy; patients who underwent radiotherapy of the head and neck region in the previous 12 months; patients with temporomandibular and/or parafunctional dysfunctions; pregnancy; poor oral hygiene or inability to undergo the follow-up protocol. The following inclusion criteria were applied for the selection of the study group: patients over the age of 18; lower total edentulous patients or patients made edentulous due to the presence of severely compromised teeth; mandibular atrophy with residual crest not less than $5 \mathrm{~mm}$ from the roof of the mandibular canal; authorization by the patient to participate in the study.

\subsection{Follow-Up Protocol}

Of the 150 patients, 40 were excluded because they were severe smokers, 12 were on bisphosphonate therapy, 41 had high risk factors, five had temporomandibular osteoarthritis, 25 were unable to follow the protocol, and eight had poor oral hygiene. The resulting sample consisted of 19 subjects, of which 11 were men aged between 62 and 77 with an average age of 69.5 and eight were women aged between 61 and 71 with an average age of 66 .

According to the Italian legal system no. 127 of 1996, these patients are counted as fragile subjects, not only due to their age, but also due to their economic status and local biological conditions. All selected patients were completely edentulous or to be rendered edentulous as the residual elements could not be used as a source of anchorage. 
The sample was subjected to preventive anamnestic tests and then underwent imaging tests for therapeutic planning.

The Ethics Committee approved the study (n. 55/2018.19), and all patients signed an informed consent form.

The sample underwent implant surgery with the use of ultrashort implants $(4 \mathrm{~mm}$ long, $4 \mathrm{~mm}$ in diameter in the premolar and canine area and $4.5 \mathrm{~mm}$ in diameter in the molar area, with the insertion torque of $60 \mathrm{Nw}$ ) Twinkon4, TEKKA, Global D. This type of implant is a grade 5 titanium alloy (TiAI6V4), sandblasted and double-etched, with a surface roughness of $1-2 \mu \mathrm{m}$.

A temporary prosthesis with immediate loading was applied to all patients, since the characteristics of the implant design and of the transmucosal type allowed no solution other than to solder the implants, as already experimented in other implant prosthetic protocols.

\subsection{Implant Characteristics and Surgical Protocol}

The implant surgery involved the use of the ultrashort implants ( $4 \mathrm{~mm}$ long) Twinkon4, TEKKA, Global D. This type of implant is a grade 5 titanium alloy (TiAI6V4), sandblasted and double-etched, with a surface roughness of 1-2 $\mu \mathrm{m}$.

All the considered patients were fitted with a temporary prosthesis with immediate loading.

The surgical protocol involved oral administration of antibiotic therapy with $2 \mathrm{~g}$ of amoxicillin $1 \mathrm{~h}$ before and then $1 \mathrm{~g}$ every $12 \mathrm{~h}$ for 5 days.

Before starting the surgery, the perioral surface was disinfected with povidone iodine (10\% Betadine), while the patients were intraorally rinsed with a $0.2 \%$ chlorhexidine solution for $60 \mathrm{~s}$. The loco-regional anesthesia was performed with $4 \%$ articaine with epinephrine 1:100,000 (CITOCARTIN “100” Molteni Dental).

The protocol included the use of a positional guide for the implant placement.

The positional guide was applied, and a punch was made on the crestal mucosa to find the positioning of the implant; then, a full thickness crestal incision was made to skeletonize the underlying bone (Figure 1).

The Tekka protocol was used for the positioning of the ultrashort implants (TwinKon4 Tekka Global D). This protocol requires the use of drill bits with increasing diameter up to the diameter necessary for the insertion of the corresponding implant. A total of 38 TwinKon 4 implants, with a $4.5 \mathrm{~mm}$ diameter, were placed in the area 3.6 and 4.6 . A total of 76 TwinKon 4 implants, with a $4 \mathrm{~mm}$ diameter, were placed in the area of the premolar and canine. The insertion torque was $60 \mathrm{~N}$.

Once the mucosa was sutured with detached or X-shaped stitches, the temporary prosthesis was fixed on the temporary abutment, taking care to make the peri-implant area easy to clean using routine oral hygiene procedures (Figure 2).

\subsection{Prostheses Project}

The operating protocol first envisaged the survey of a conventional analogue impression in alginate (Kromopan Lascod s.p.a) of the dental arches; then, an articulation base was built for the detection of the neutral space and for the vertical dimension. This method used TENS (Trans Cutaneous Electrical Nerve Stimulation) stimulation (J5 Myomonitor ${ }^{\circledR}$ TENS Unit device of Myotronics-Noromed, Inc., Tukwila, WA, USA) with electrodes (Myotrode SG Electrodes ${ }^{\circledR}$, Myotronics-Noromed, Inc., Tukwila, WA, USA), whereas resin was used for the detections (Sapphire Resin, Myoprint) (Figure 3).

Subsequently, both a conventional prosthesis and a baryta resin duplicate were made to perform the cone beam.

In order to find the implant position, the DICOM (Digital Imaging and Comunications in Medicine) images were processed with the acquisition program (3 shape Implant Studio) to create a positional template for the surgical phase.

The implant position was guided by a functional gnathological evaluation and by a series of prosthetic reference factors such as the distribution of the occlusal load and the 
neutral space (which is the space where the resultant of the strength of the muscles, tongue, and cheeks is equal to zero).
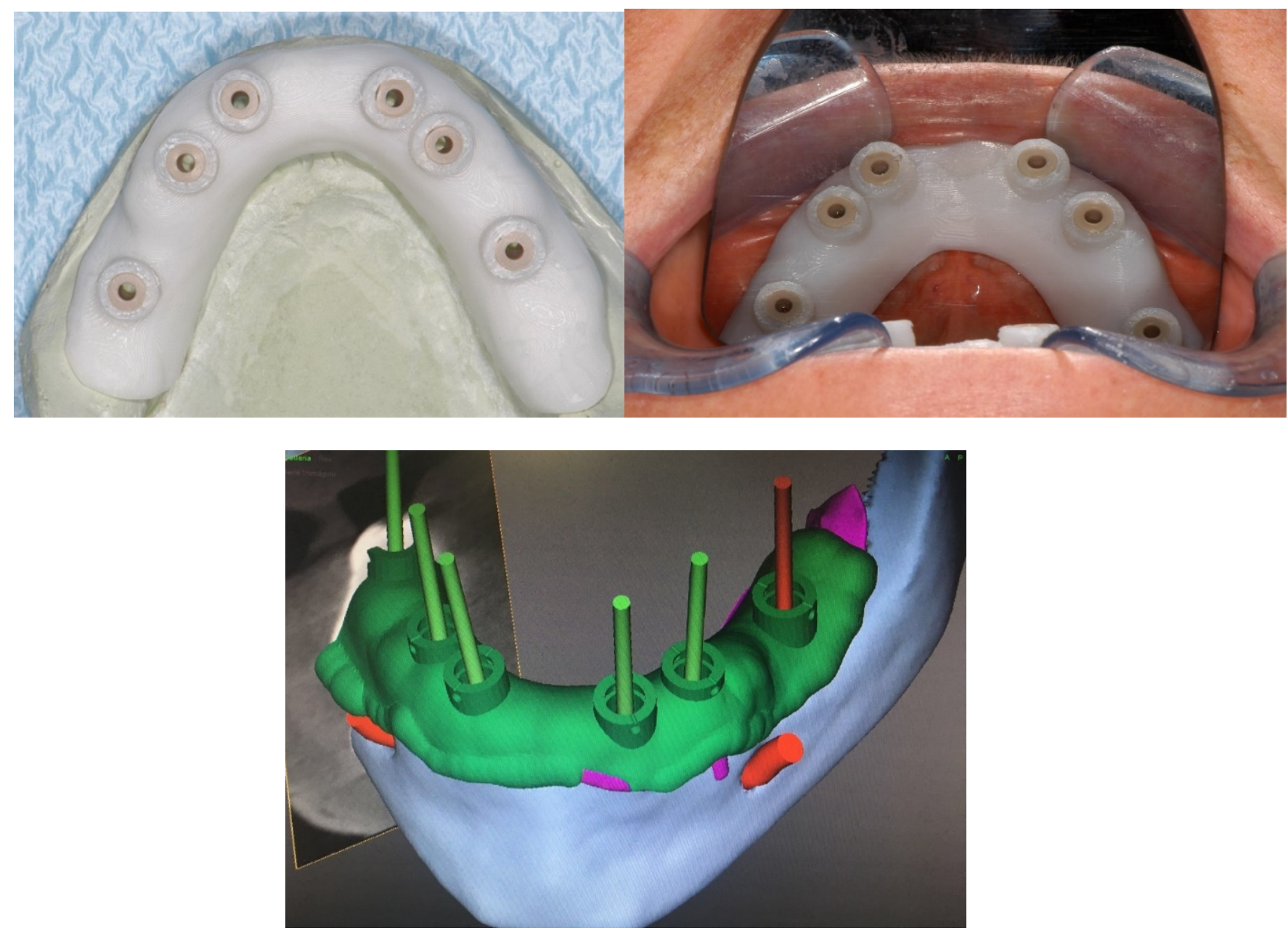

Figure 1. Positional guide for the implant placement.

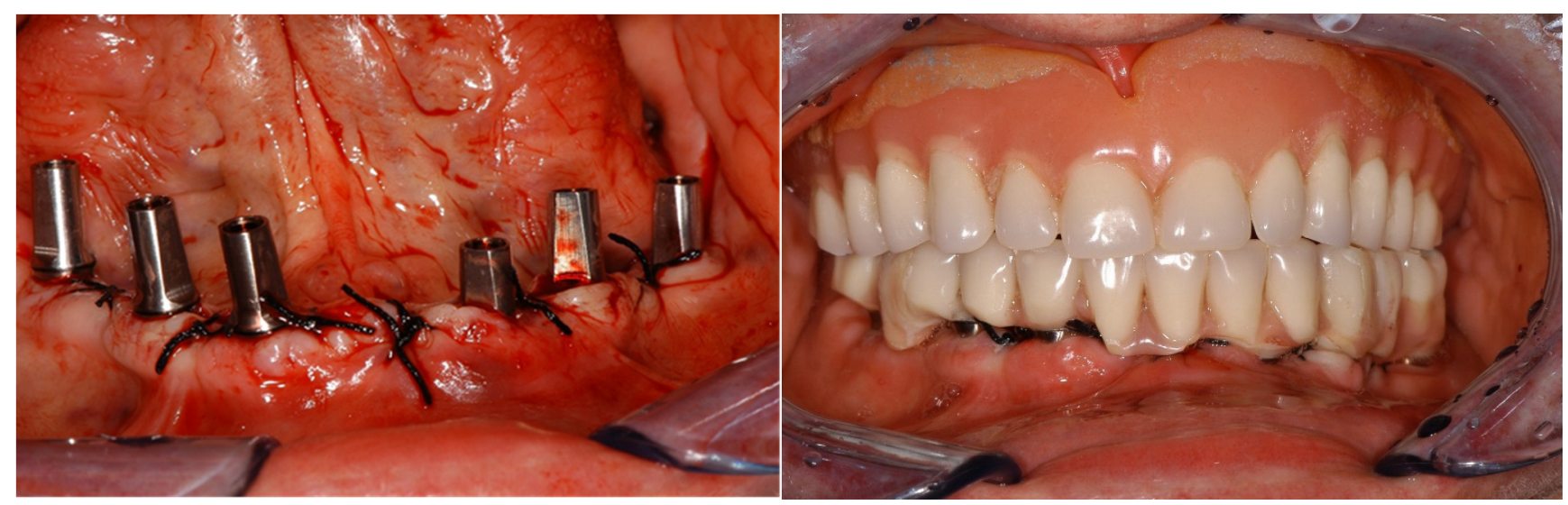

Figure 2. The temporary prosthesis was fixed on the temporary abutment. 

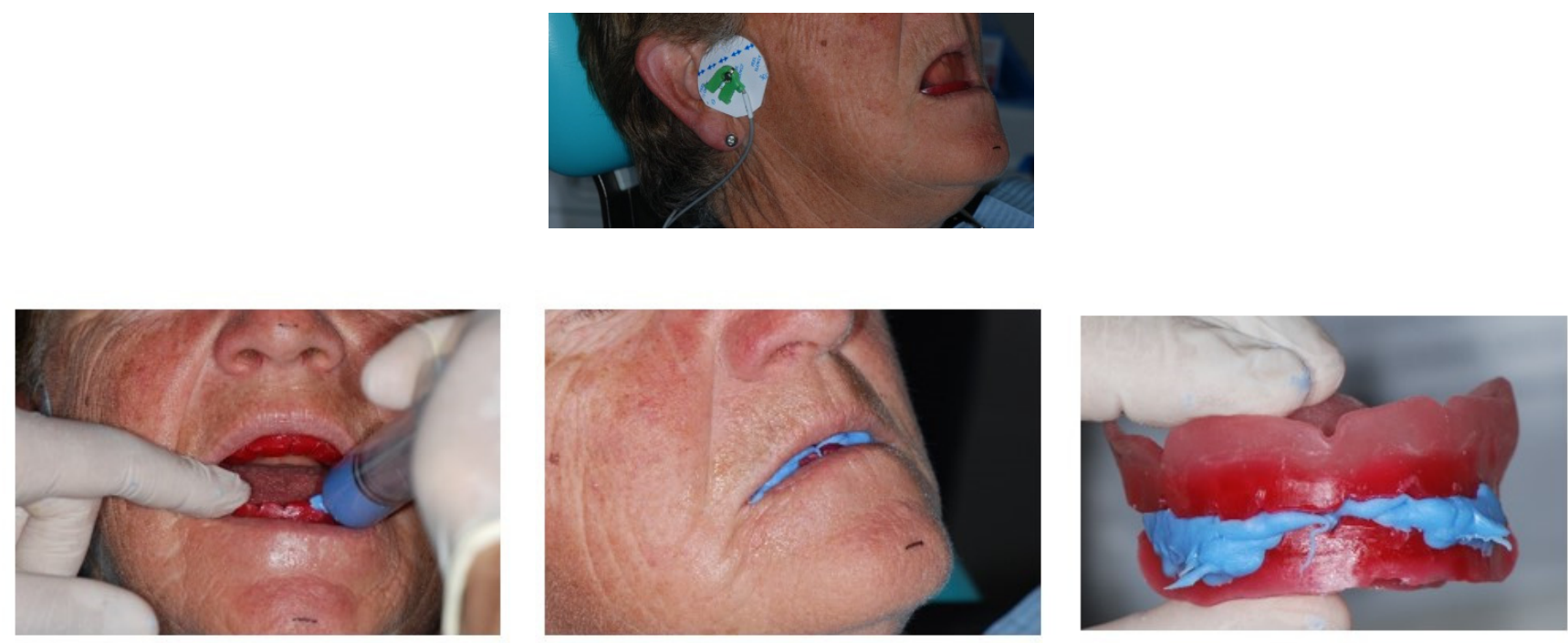

Figure 3. TENS (Trans Cutaneous Electrical Nerve Stimulation) stimulation with electrodes and detection with Myopront resin.

\subsection{Follow-Up}

Seven days after surgery, a clinical examination of the patient was performed, in which the healing status of the mucosa and the implant stability were analyzed and the sutures were removed. After 4 months, a cone beam was performed to assess whether the bone healing status had no problems (Figure $4 \mathrm{~b}$ ), before proceeding with the realization of the final prosthesis with a screwed bar. An OPG (Orthopantomography) was carried out 1 year (Figure 4c) and an X-ray OPG was carried out 3 years (Figure 4d) after the realization of the definitive prosthesis.

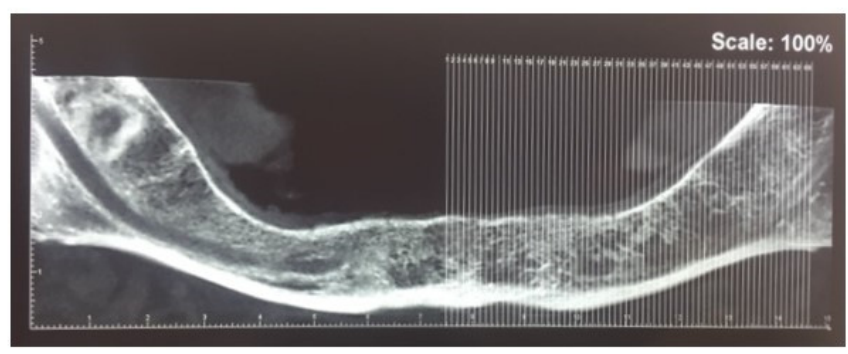

a.

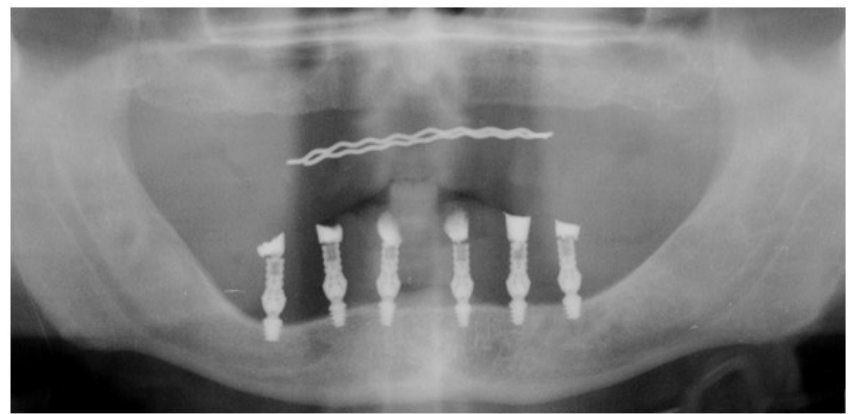

c.

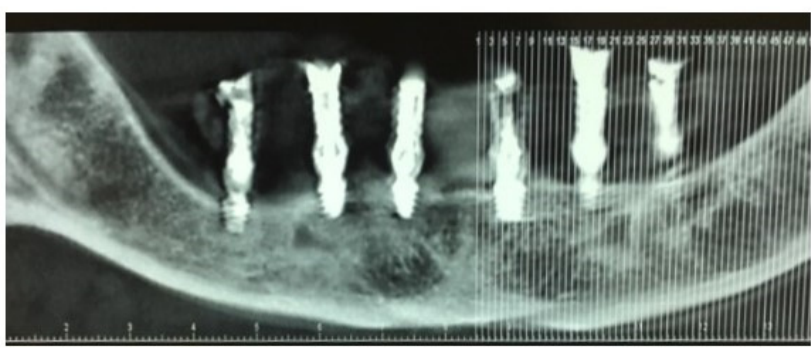

b.

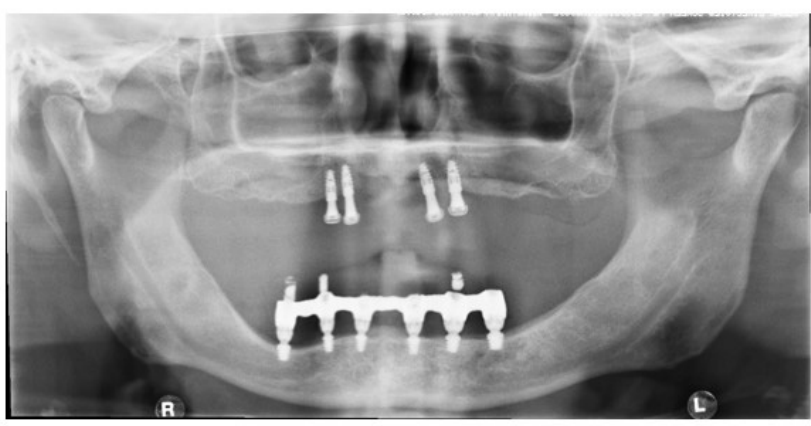

d.

Figure 4. Radiographic checks: (a) cone beam before surgery; (b) cone beam 4 months after surgery; (c) OPG (Orthopantomography)1 year later; (d) OPG 3 years later. 


\section{Statistical Analysis}

The primary outcome was the number of patients who, at the 3 year follow-up, had a stable prosthesis supported by at least four implants, evaluated using chi-square test. The secondary outcome was the implant survival at T1, T2, and T3, statistically evaluated using the Fisher exact test, and the endpoint was the survival rate of the implants at T4. Any statistically differences were considered significant at a $p$-value $<0.05$.

\section{Results}

Nineteen patients were included in the retrospective analysis, 11 males and eight females, with an age ranging from 64 to 77 . Implants were mostly lost 1 week after loading and at the fourth month follow-up.

\subsection{Primary Outcome Results}

Eighteen patients at $\mathrm{T} 4$ presented a stable prothesis supported by $\geq 4$ implants. As shown in Table 1, the chi-square test showed a statistically significant difference $(p<0.05)$.

Table 1. The significant majority of the patients at the 3 year follow-up presented a stable and functional implant-supported prothesis. The chi-square test showed the significant success of the proposed protocol.

\begin{tabular}{cccc}
\hline \multicolumn{4}{c}{ Primary Outcome: Success of the Prothesis } \\
\cline { 1 - 3 } Prothesis Outcome & Frequency $(N)$ & Percentage $(\%)$ & \\
\hline Successful prosthesis & 18 & 5.26 & $<0.05$ \\
\hline Unsuccessful prosthesis & 1 & 94.73 & \\
\hline
\end{tabular}

\subsection{Secondary Outcome Results}

The Fisher exact test applied to determine the implant loss occurrence at the considered follow-up periods (T1, T2, T3) showed a statistically significant difference (Table 2).

Table 2. The Fisher test was significant with a $p$-value $<0.05$ regarding the time of implant loss occurrence.

\begin{tabular}{cccc}
\hline Implant Loss Occurrence & T1 (1 Week) & T2 (4 Months) & T3 (12 Months) \\
\hline Loss & $3(15.79 \%)$ & $7(36.84 \%)$ & $0(0)$ \\
\hline No Loss & $16(84.21 \%)$ & $12(83.16 \%)$ & $19(100 \%)$ \\
\hline
\end{tabular}

\subsection{Endpoint}

At T4, the survival rate of the implants was $85 \%$, with a loss of 16 implants from a total of 114 implants.

\section{Discussion}

The retrospective follow-up of patients who underwent full-arch rehabilitation with ultrashort implants showed how the critical points of rehabilitation were the first week and the 4 months after implant placement and the related prosthetic loading, as observed in the literature [4].

Overall, the unsuccess of the rehabilitation was not frequent, and basically only one patient could not rely on this type of prosthetic rehabilitation.

If the surgical intervention on jawbones is considered as a minor type on surgery and with a very low risk, the diagnosis and planning stages are fundamental for rehabilitation, which can be comfortable and satisfying for the patients [16].

The oral cavity, with its sensitivity and peculiar functionality due not only to its occlusion and masticatory function but also to its phonatory, respiratory, and tasting function, requires a careful rehabilitation plan [18]. 
Currently, the rehabilitation of small edentulism requires an established plan, facilitated by the presence of other dental elements. Edentulism cases requiring a complete denture rehabilitation are challenging for both anatomical and functional reasons [21]. Indeed, the bone availability is conditioned by the degree of alveolar resorption, which places the noble structures such as the inferior alveolar nerve and the maxillary sinus at risk [22]. In addition, risks of fracture are higher in cases of severely atrophic jaws during surgical intervention [21].

The loss of the vertical dimension modifies the functionality of the masticatory organ, and spotting the neutral zone might be difficult [16].

Several surgical techniques are available to improve the volumetric bone availability. Vertical ridge augmentation, large sinus lift, xenograft interventions, and placing of pterygoid implants or zygomatic implants are some of the most famous and new techniques proposed in the literature [2]. However, the high cost and the morbidity risks associated with these techniques are not appealing from both the clinician's and the patient's point of view $[10,13,23,24]$.

The improvement of implant surfaces and the establishment of defined surgical protocols allowed short implants to be a reliable alternative to rehabilitate small edentulism in atrophic jaws; however, the rehabilitation of full edentulism using full-arch prostheses has also been reported with a certain degree of success.

Overall, the primary outcome showed the success of the ultrashort implant-supported prosthetic protocol. However, the rate of implant loss was slightly higher, if compared with the survival rate reported by the Group 1 ITI Consensus Report, which reported a survival rate ranging from $86.7 \%$ to $100 \%$ [13]. However, the reported data included two different variables which were not considered or studied in the literature: the loading time (immediate) and the type of prothesis (full-arch rehabilitation).

The available studies in the literature reported the survival rate of short and ultrashort implants with immediate or delayed loading supporting single crowns [23-27].

On the other hand, few case reports are available in the literature reporting full-arch prothesis supported by ultrashort implants and placed with immediate loading $[17,19,28]$.

Indeed, Falisi et al. reported a full-arch rehabilitation on ultrashort implants with no postoperative complication and success of the prosthesis integration, in functional and aesthetic terms. In this case, the prosthesis was planned according the neutral zone of the patient [23].

Pistilli et al. also reported the case of a rehabilitation of a severe atrophic mandible on implants no longer than $4 \mathrm{~mm}$, highlighting how this therapeutical choice reduced timings, costs, and postoperative complications. In this case, the occlusal loading was the most balanced [21].

However, the data of a previous study showed how immediate loading can affect the survival rate of implants but not the final prothesis success, showing how the correct distribution of the occlusal forces in the period after implant insertion can positively influence the final outcome [16].

Indeed, the healing period after the surgical stage is the most crucial one, since the bone tissue is submitted to a remodeling process balanced between the resorption and production of the mineralized tissue [25].

The theme of immediate loading on short implants is still under investigation among scientists and clinicians. Indeed, Weerapong et al., on the basis of the results of their randomized clinical trial, stated that immediate loading on short implants gives survival rates comparable to immediate loading on longer implants, together with the use of new technologies and CAD/CAM (Computer Aided Design/Computer Aided Manufacturing) replanning of the prosthetic device [26].

Within the limits of the current study, regarding the sample size and the observational nature of the study, the full-arch rehabilitation with immediate loading on ultrashort implants showed good results with few postoperative complications and related low biological cost. 
Future prospective studies and randomized clinical trials with a larger sample size are needed to evaluate the survival rate of full-arch rehabilitation on short and ultrashort implants with appropriate occlusal loading.

\section{Conclusions}

Results from our study suggest the adequate clinical performance of short implants after 3 years of immediate loading. Thus, $4 \mathrm{~mm}$ ultrashort implants with a diameter of 4 and $4.5 \mathrm{~mm}$ could represent a good alternative to rehabilitate edentulous atrophic jaws or jaws with residual elements that cannot be used as a source of anchorage.

\section{Ethics}

The study was approved by the Internal Review Board of University of L'Aquila, $\mathrm{n}$. $55 / 2018.19$.

Author Contributions: Conceptualization, G.F. and C.R.; methodology, G.F.; validation, C.D.P., investigation, G.B., S.R., C.F.; resources, G.F. and R.G.; data curation, G.B.; writing-original draft preparation, G.B. and S.R.; writing - review and editing, G.F. and R.G. All authors have read and agreed to the published version of the manuscript.

Funding: Biomedic s.r.l. Via Fiumedinisi 62 Rome, 00133 Italy.

Institutional Review Board Statement: The study was conducted according to the guidelines of the Declaration of Helsinki, and approved by the Internal Review Board of University of L'Aquila, $n$. 55/2018.19.

Informed Consent Statement: Informed consent was obtained from all subjects involved in the study.

Data Availability Statement: Data will be availble upon resonable request to the corresponding author.

Acknowledgments: The authors are grateful to Sara Bernardi for data, critical discussions, and advice.

Conflicts of Interest: The authors declare no conflict of interest.

\section{References}

1. Yalçın, M.; Can, S.; Akbaş, M.; Dergin, G.; Garip, H.; Aydil, B.; Varol, A. Retrospective Analysis of Zygomatic Implants for Maxillary Prosthetic Rehabilitation. Int. J. Oral Maxillofac. Implant. 2020, 35, 750-756. [CrossRef]

2. DENTAL SUPPLEMENT; Scarano, A.; Bernardi, S.; Rastelli, C.; Mortellaro, C.; Vittorini, P.; Falisi, G. Soft tissue augmentation by means of silicon expanders prior to bone volume increase: A case series. J. Biol. Regul. Homeost Agents 2020, 33, 77-84.

3. Srinivasan, M.; Vazquez, L.; Rieder, P.; Moraguez, O.; Bernard, J.-P.; Belser, U.C. Survival rates of short (6 mm) micro-rough surface implants: A review of literature and meta-analysis. Clin. Oral Implant. Res. 2013, 25, 539-545. [CrossRef] [PubMed]

4. Bernardi, S.; Gatto, R.; Severino, M.; Botticelli, G.; Caruso, S.; Rastelli, C.; Lupi, E.; Roias, A.Q.; Iacomino, E.; Falisi, G.; et al. Short Versus Longer Implants in Mandibular Alveolar Ridge Augmented Using Osteogenic Distraction: One-Year Follow-up of a Randomized Split-Mouth Trial. J. Oral Implant. 2018, 44, 184-191. [CrossRef] [PubMed]

5. Perelli, M.; Abundo, R.; Corrente, G.; Saccone, C. Short (5 and $7 \mathrm{~mm}$ long) porous implants in the posterior atrophic maxilla: A 5-year report of a prospective single-cohort study. Eur. J. Oral Implant. 2012, 5, 265-272.

6. Rossi, F.; Botticelli, D.; Cesaretti, G.; De Santis, E.; Storelli, S.; Lang, N.P. Use of short implants (6 mm) in a single-tooth replacement: A 5-year follow-up prospective randomized controlled multicenter clinical study. Clin. Oral Implant. Res. 2016, 27, 458-464. [CrossRef]

7. Esposito, M.; Barausse, C.; Pistilli, R.; Checchi, V.; Diazzi, M.; Gatto, M.R.; Felice, P. Posterior jaws rehabilitated with partial prostheses supported by $4.0 \times 4.0 \mathrm{~mm}$ or by longer implants: Four-month post-loading data from a randomised controlled trial. Eur. J. Oral Implant. 2015, 8, 221-230.

8. Ewers, R. The incisal foramen as a means of insertion for one of three ultra-short implants to support a prosthesis for a severely atrophic maxilla-A short-term report. Heliyon 2018, 4, e01034. [CrossRef] [PubMed]

9. Elias, D.; Valerio, C.; De Oliveira, D.; Manzi, F.; Zenóbio, E.; Seraidarian, P. Evaluation of Different Heights of Prosthetic Crowns Supported by an Ultra-Short Implant Using Three-Dimensional Finite Element Analysis. Int. J. Prosthodont. 2020, 33, 81-90. [CrossRef]

10. Felice, P.; Barausse, C.; Pistilli, V.; Piattelli, M.; Ippolito, D.R.; Esposito, M. Posterior atrophic jaws rehabilitated with prostheses supported by $6 \mathrm{~mm}$ long $\times 4 \mathrm{~mm}$ wide implants or by longer implants in augmented bone. 3-year post-loading results from a randomised controlled trial. Eur. J. Oral Implant. 2018, 11, 175-187. 
11. Sierra-Sánchez, J.-L.; García-Sala-Bonmatí, F.; Martínez-González, A.; García-Dalmau, C.; Mañes-Ferrer, J.-F.; Brotons-Oliver, A. Predictability of short implants $(<10 \mathrm{~mm})$ as a treatment option for the rehabilitation of atrophic maxillae. A systematic review. Med. Oral Patol. Oral Cir. Bucal 2016, 21, e392-e402. [CrossRef]

12. Sgolastra, F.; Petrucci, A.; Severino, M.; Gatto, R.; Monaco, A. Smoking and the risk of peri-implantitis. A systematic review and meta-analysis. Clin. Oral Implant. Res. 2015, 26, e62-e67. [CrossRef] [PubMed]

13. Jung, R.E.; Al-Nawas, B.; Araujo, M.; Avila-Ortiz, G.; Barter, S.; Brodala, N.; Chappuis, V.; Chen, B.; De Souza, A.; Almeida, R.F.; et al. Group 1 ITI Consensus Report: The influence of implant length and design and medications on clinical and patient-reported outcomes. Clin. Oral Implant. Res. 2018, 29, 69-77. [CrossRef] [PubMed]

14. Falisi, G.; Severino, M.; Rastelli, C.; Bernardi, S.; Caruso, S.; Galli, M.; Lamazza, L.; Di Paolo, C. The effects of surgical preparation techniques and implant macro-geometry on primary stability: An in vitro study. Med. Oral Patol. Oral Cir. Bucal 2017, 22, e201-e206. [CrossRef]

15. Porwal, A.; Sasaki, K. Current status of the neutral zone: A literature review. J. Prosthet. Dent. 2013, 109, 129-134. [CrossRef]

16. Frascaria, M.; Pietropaoli, D.; Casinelli, M.; Cattaneo, R.; Ortu, E.; Monaco, A. Neutral zone recording in computer-guided implant prosthesis: A new digital neuromuscular approach. Clin. Exp. Dent. Res. 2019, 5, 670-676. [CrossRef]

17. Lollobrigida, M.; Maritato, M.; Bozzuto, G.; Formisano, G.; Molinari, A.; De Biase, A. Biomimetic Implant Surface Functionalization with Liquid L-PRF Products: In Vitro Study. Biomed. Res. Int. 2018, 8, 9031435. [CrossRef]

18. Tettamanti, L.; Andrisani, C.; Bassi, M.A.; Vinci, R.; Silvestre-Rangil, J.; Tagliabue, A. Immediate loading implants: Review of the critical aspects. Oral Implantol. 2017, 10, 129-139. [CrossRef]

19. Cicconetti, A.; Passaretti, A.; Rastelli, C.; Rastelli, E.; Falisi, G. Innovations in oral and maxillofacial surgery: Biomimetics meets physiology. J. Biol. Regul. Homeost Agents 2019, 33, 1609-1613. [PubMed]

20. Schiffman, E.; Ohrbach, R.; Truelove, E.; Look, J.; Anderson, G.; Goulet, J.P.; List, T.; Svensson, P.; Gonzalez, Y.; Lobbezoo, F.; et al. Diagnostic Criteria for Temporomandibular Disorders (DC/TMD) for Clinical and Research Applications: Recommendations of the International RDC/TMD Consortium Network* and Orofacial Pain Special Interest Group. J. Oral Facial Pain Headache 2014, 28, 6-27. [CrossRef]

21. Pistilli, R.; Barausse, C.; Gasparro, R.; Berti, C.; Felice, P. Minimally Invasive Fixed Rehabilitation of a Totally Edentulous Severely Atrophic Mandible with 4-mm Ultrashort Immediately Loaded Implants: A Case Report. Int. J. Periodontics Restor. Dent. 2020, 40, 549-559. [CrossRef] [PubMed]

22. Bernardi, S.; Mummolo, S.; Ciavarelli, L.M.; Vigni, M.L.; Continenza, M.A.; Marzo, G. Cone beam computed tomography investigation about the antral artery anastomosis in a center of Italy population. Folia Morphol. 2016, 75, 149-153. [CrossRef]

23. Falisi, G.; Bernardi, S.; Rastelli, C.; Pietropaoli, D.; De Angelis, F.; Frascaria, M.; Di Paolo, C. "All on short" prosthetic-implant supported rehabilitations. Oral Implantol. 2017, 10, 477-487. [CrossRef] [PubMed]

24. AlZarea, B.K. Oral health related quality-of-life outcomes of partially edentulous patients treated with implant-supported single crowns or fixed partial dentures. J. Clin. Exp. Dent. 2017, 9, e666-e671. [CrossRef] [PubMed]

25. Barndt, P.; Zhang, H.; Liu, F. Immediate loading: From biology to biomechanics. Report of the Committee on Research in Fixed Prosthodontics of the American Academy of Fixed Prosthodontics. J. Prosthet. Dent. 2015, 113, 96-107. [CrossRef]

26. Weerapong, K.; Sirimongkolwattana, S.; Sastraruji, T.; Khongkhunthian, P. Comparative Study of Immediate Loading on Short Dental Implants and Conventional Dental Implants in the Posterior Mandible: A Randomized Clinical Trial. Int. J. Oral Maxillofac. Implant. 2019, 34, 141-149. [CrossRef] [PubMed]

27. Bolle, C.; Felice, P.; Barausse, C.; Pistilli, V.; Trullenque-Eriksson, A.; Esposito, M. 4 mm long vs longer implants in augmented bone in posterior atrophic jaws: 1-year post-loading results from a multicentre randomised controlled trial. Eur. J. Oral Implantol. 2018, 11, 31-47.

28. Felice, P.; Karaban, M.; Pistilli, R.; Bellini, P.; Bonifazi, L.; Barausse, C. Minimally invasive rehabilitation of a severely atrophic and fully edentulous maxilla using 4-mm-ultrashort implants: A case report with 1-year follow-up. Oral Maxillofac. Surg. Cases 2020, 6, 100176. [CrossRef] 